\title{
The Potential of Algae Spyrogyra peipingensis to Produce Nanogold in Batch Culture Medium
}

\author{
Agusrahman Ekaputra Abas ${ }^{1}$, Chitriani Armidha ${ }^{2}$, Arbaina Syahdinnur ${ }^{1}$ \\ ${ }^{1}$ Department of Biology, Faculty of Mathematics and Natural Sciences, Hasanuddin \\ University, Makassar, Indonesia \\ ${ }^{2}$ Department of Chemistry, Faculty of Mathematics and Natural Sciences, Hasanuddin \\ University, Makassar, Indonesia
}

\begin{abstract}
The biosynthesis of gold nanoparticles by using algae Spirogyra peipingensis was conducted. This research aimed to determine biosynthesis of gold nanoparticles by using algae Spirogyra peipingensis with different concentration and incubation time. The synthesis of gold nanoparticles using $\mathrm{HauCl}_{4}$ solution with variations of concentration 5 ppm, $10 \mathrm{ppm}, 15 \mathrm{ppm}$ and $20 \mathrm{ppm}$ respectively in 1 liter of aquabidest. Algae Spirogyra peipingensis was grown in $\mathrm{HauCl}_{4}$ medium with the addition of 0.2 gr Sulfahri-01 nutrient. Then each 5 gram Spirogyra algae incubated in $\mathrm{HAuCl}_{4}$ medium with the addition of Sulfahri-01 0.2 gram nutrients. 4 hours long incubation under exposure to sunlight. Nanoparticle size determination is done by looking at the color that appears in the solution. From the results of the research, it is known that Spirogyra peipingensis algae is able to synthesize gold nanoparticles characterized by the color change in algae biomass from green to purple color after being treated and forming gold nanoparticles with size 40-60 $\mathrm{NM}$. The best-used $\mathrm{HAuCl} 4$ consent is $5 \mathrm{ppm}$ with the smallest particle size.
\end{abstract}

\section{Article History}

Received 8 June 2018

Accepted 14 July 2018

Keyword

Intracelullar, Spirogyra

peipingensis,

$\mathrm{HAuCl}_{4}$,

Biosynthesis.

\section{Introduction}

The development of nanotechnology applications has driven the industrial revolution globally. It has been reported that more than 400 companies worldwide have undertaken nanotechnology research and development activities. As a relatively new discipline, globally nanotechnology research and applications are growing very rapidly (Hoerudin \& Irawan, 2015). Nanoparticles are a scope of nanotechnology. It is called a nanoparticle when the particle size is in the range of 1-100 NM. With unique properties and sizes, nanoparticles have great potential as a material that is superior in the future. The nanoparticle material can be organic material such as 2.5 NM DNA, 50 NM protein, $100 \mathrm{NM}$ Virus type (Pantidos \& Horsfall, 2014), or inorganic materials such as semi-conductor 
nanoparticles (titanium oxide and zinc oxide), and precious metal nanoparticles (silver and gold) (Shaileyee \& C Mandal, 2014).

According to Rohiman et al (2014), in the world of nanotechnology, research on gold nanoparticles has attracted the attention of many world researchers because of its superiority in its various applications. Gold nanoparticles (AuNPs) are gold ions that are reduced to non-charged gold and nano-sized (Maruani, 2013). AuNPs have prominent advantages compared to gold particles, which have optical and electronic properties with low toxicity (Rohiman, et al, 2014) In addition, AuNPs can be used as a water purifier (Qian, et al, 2013), carriers of cancer-killing drugs (Alhalili, et al, 2017), chemical and biological sensors (Qin, et al, 2018), catalysts (Amdouni, et al, 2018), biohydrogen production and various biomedical applications (Zhang \& Shen, 2007). The production of AuNPs can be carried out by various chemical methods, but the production of eco-friendly nanoparticles are being intensively developed (Sovawi, et al., 2016) through biosynthetic pathways using biological agents such as yeast, fungi, bacteria, plants, plant extracts and algae (Thakkar, 2010).

Algae are thallophyta organisms that have been known to have a lot of potential. One of the most easily found algae in Indonesia is Spirogyra peipingensis algae. This algae has the potential to reduce both organic and inorganic compounds. Based on the Bahri (2017) study, Spirogyra pepingensis algae can significantly reduce toxicity, absorbing heavy metals (Gupta \& Rastogi, 2008), and reducing textile waste (Özer, et al., 2006) that have mechanisms such as activated carbon. The use of algae as a gold metal bioreductor has previously been done by Roychordhury (2016). Algae used derived from the group of prokaryotic algae (Cyanobacteria) namely Anabaena sphaerica and eukaryotic green algae namely Chlorococcum infusionum. The alignment of cell components in algae such as carotenoids, polysaccharides, proteins and pigments in chloroplasts / thylakoids has a major role as reducing agents in the biosynthesis of nanoparticle metals (Roychordhury, et al., 2016). carotenoids, polysaccharides, proteins and chloroplast pigments are also found in Spirogyra algae (Tipnee, 2015). Therefore, Spirogyra peipingensis algae is believed to have the potential to reduce gold particles to nano-sized gold particles.

\section{Materials and Methods}

\section{Algae Preparation}

Alga Spirogyra peipingensis obtained from the rice field area around the city of Makassar. then the algae is cleaned and identifiable under a light microscope before being cultured on growth medium sulfahri-01 (3000 lux, 12:12) which later becomes algae stock during the research phase.

\section{Preparation of $\mathrm{HAuCl}_{4}$ Solution}

0.1 grams of gold metal dissolved into $20 \mathrm{ml}$ aquaregia ( $3 \mathrm{HCl}: 1 \mathrm{HNO} 3)$. heat over hot plate until completely dissolved. then dilute it into a $500 \mathrm{ml}$ measuring flask to make a parent solution of $200 \mathrm{ppm}$. The $\mathrm{HAuCl} 4$ gold mains solution was diluted with aquabides with variations in dilution concentration of $5 \mathrm{ppm} / \mathrm{L}, 10 \mathrm{ppm} / \mathrm{L}, 15 \mathrm{ppm} / \mathrm{L}, 20 \mathrm{ppm} / \mathrm{L}$ in $1000 \mathrm{ml}$ aquarium. 


\section{Incubation of algae Spirogyra peipingensis into gold medium}

$5 \mathrm{gr}$ of Spirogyra peipingensis biomass were incubated on $\mathrm{HAuCl} 4$ gold medium and $0.2 \mathrm{gr}$ of additional surlfahri-01 nutrient media under exposure to 4 hours of sunlight. The top of the aquarium is covered with a cling wrap that is sufficiently ventilated for air circulation.

\section{Determines the size of gold nanoparticles}

After Spirogyra peipingensis algae incubation for 4 hours, the yellowish amorphous media solution was changed. The color variations formed can be used to determine the particle size of the synthesized product. This refers to the color guide of the solution to determine the size of the gold nanoparticles published by Noutarianni (2013).

\section{Results and discussion}

\section{Algae Preparation}

Fresh algae biomass Spirogyra peipingensis taken in the rice fields Sudiang, Makassar, Indonesia. then cleaned and identified under a magnification light microscope 100 times above Sedgwick Rafter Counting Chamber (SRC). Determination of Spirogyra peipingensis algae type was based on the publication (Zarina, et al, 2007) with unbranched filamentous morphology, with vegetative cell width 104-157 $\mu \mathrm{m}$ and length 156-200 $\mu \mathrm{m}$ and 5-7 pyrenoid. After identification, then Alga Spirogyra peipingensis in culture in $0.2 \mathrm{gr} / \mathrm{L}$ medium sulfahri-01 with water height $20 \mathrm{~cm}$ at 3000 lux lighting lamp with a duration of 12:12. The composition of the media has been tested based on Sulfahri, et al, (2016). From several variations of water height $(10,20,30 \mathrm{~cm}), 20 \mathrm{~cm}$ is the most ideal water level with the highest level of productivity.

\section{Algae Incubation}

$5 \mathrm{gr}$ Biomass Algae Spirogyra peipingensis was incubated in a medium composed of $0.2 \mathrm{gr} / \mathrm{L}$ sulfahri-01 in several concentrations of $\mathrm{HAuCl} 4$ with a series of $5 \mathrm{ppm}, 10 \mathrm{ppm}, 15$ ppm and $20 \mathrm{ppm}$. Each solution was placed in a $20 \mathrm{~cm}$ glass tank with a capacity of $1000 \mathrm{ml}$ and placed under 4 hours of sun exposure. The sulfahri-01 medium is the most superior medium among several types of mediums compared to having high nitrogen content which may play an important role (Sulfahri, et al, 2016)

After incubation for 4 hours, a change of color occurs in Spirogyra peipingnsis biomass. The algae biomass, which was initially light green, became dark purple and was confirmed to have died from exposure to the metal mixture in its incubation medium (Fig.2a,2b). This change indicates that algae Spirogyra peipingensis can positively synthesize. This is in accordance with the results obtained by parial, et al (2012) who trace the types of Phormidium valderianum algae, $P$. tenue, Microcoleus chthonoplastes, Rhizoclonium fontinale and Ulva intestinalis. These five algae can positively synthesize intracellular gold nanoparticles. According to parial, et al, (2012) the five algae potentially as "Bionanofactories" and this is evident from the visual transformation of the algal thalli from green to deep purple. 


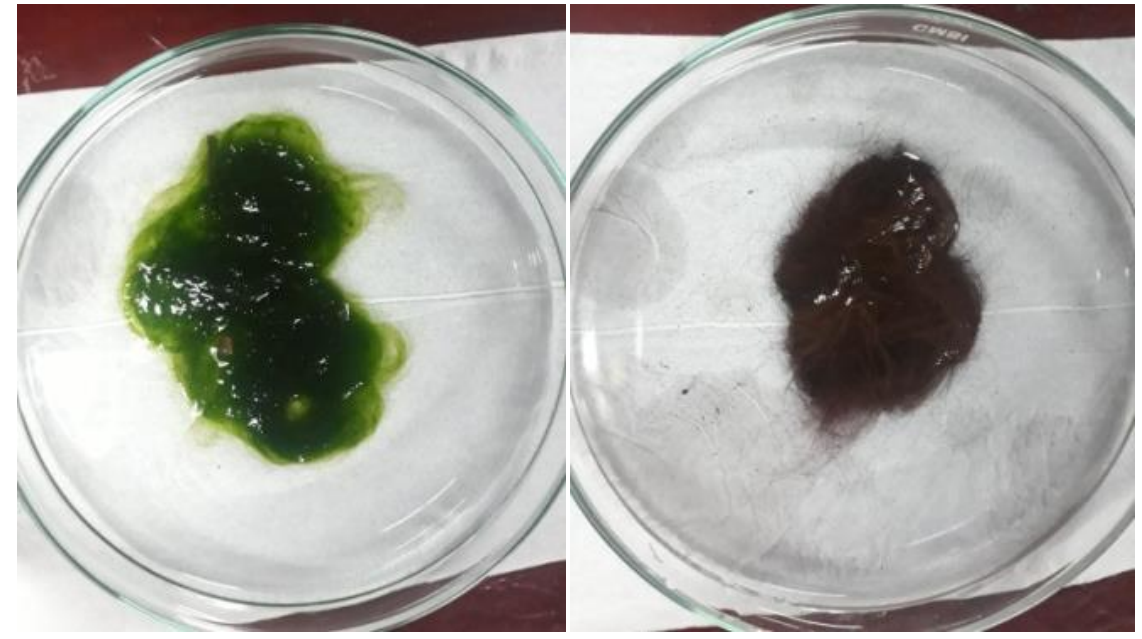

Fig. 2a. Before Incubation

Fig. 2b. After Incubation

The ability of algae as a reducing agent to synthesize gold nanoparticles is much in play by the variety of cellular components such as carotenoids, polysaccharides, proteins and complete pigments in chloroplasts / tilakoid (Royconduri, et al, 2016). The reduction of the metal ions occurs upon the surface of the algal cell as well as on the cytoplasmic membrane, leading to the formation of gold nanoparticles (Senapati, et al, 2012). The exact process of intracellular formation of gold nanoparticles by alga biomass is not yet fully understood (Parial, et al, 2012).

\section{Determines the size of gold nanoparticles}

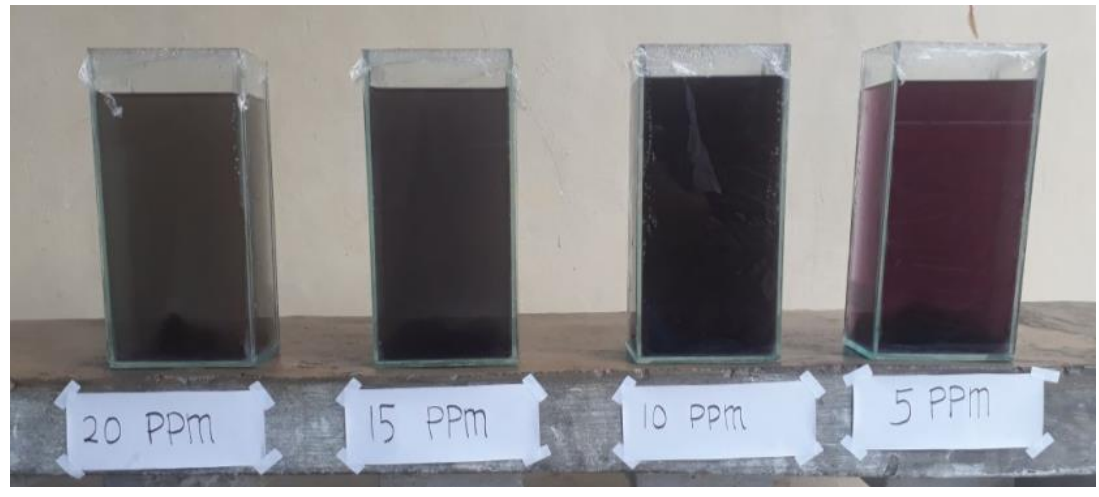

Fig. 3a. variations colour of media solutions

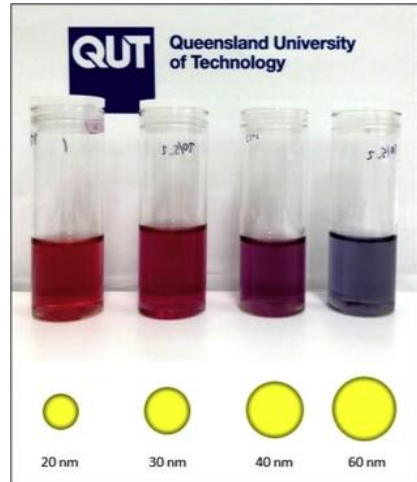

3b. GNPs sizing guide

In this way (Fig. 3a), it is possible to deduce the size of the Au NPs produced, ranging from 40-60 nm. This refers to the GNPs sizing guide based on the color of the solution (Fig.3b) from Queensland University of Technology (Notarriani, 2014). From this research, it can be seen that the bigger $\mathrm{HAuCl}_{4}$ concentration, then the size of GNPs in the file will be greater because the particle size generated in the concentration series 5,10,15,20 ppm respectively contribute the change of color dry awaiting young to blue, which describes the existence the addition of the diameter of the nanoparticles, therefore, the $5 \mathrm{ppm}$ concentration is considered the best as an incubation medium in producing gold nanoparticles.

According to Parial (2012), All applications of gold (or other) nanoparticles thus demand a well-defined size and accurate measurement of these parameters is correspondingly important. The optical property of surface plasmon resonance is directly 
dependent on the size of the gold nanoparticle: $20 \mathrm{~nm}$ particles show an orange-red colour but the colour gradually shifts to blue when particle size increases to $\sim 100 \mathrm{~nm}$. Plasmon resonance also changes with aspect ratio.

\section{Conclusions}

From the study, it can be concluded that Spirogyra peipingensis algae can synthesize gold nanoparticles. Incubation of $\mathrm{HAuCl}_{4}$ medium $5 \mathrm{ppm} 0.2 \mathrm{~g} / \mathrm{L}$ Sulfahri-01 best produces gold nanoparticles. From the color of the solution obtained, can also be concluded the smaller the concentration of $\mathrm{HAuCl}_{4}$ the smaller the size of the particles produced. The intracellular gold nanoparticles biosynthesis has great potential to develop to industrial scale because it is easier, cheaper, and environmentally friendly.

\section{Acknowledgment}

The authors gratefully acknowledge financial support from the Ministry of Research, Technology and Higher Education of Indonesia with project PKM (Program Kreativitas mahasiswa).

\section{References}

Alhalili, Z., Figueroa, D., Johnston, M. R., Shapter, J., \& Sanderson, B. 2017. Effect of Modification Protocols on the Effectiveness of Gold Nanoparticles as Drug Delivery Vehicles for Killing of Breast Cancer Cells. Australian Journal of Chemistry. 69(12) : 1402-1412.

Amdouni, S., Cherifi, Y., Coffinier, Y., Addad, A., Zaïbi, M. A., Oueslati, M., \& Boukherroub, R. 2018. Gold nanoparticles coated silicon nanowires for efficient catalytic and photocatalytic applications. Materials Science in Semiconductor Processing. 75 : 206213.

Bahri, S., Kamsinar, Sarioja, Edi, T., Nurul, F. 2017. Application of Cigeratte Filter from Algae Spyrogira peipingensis to Reduce Nicotine, Tar, and Carbon Monoxide. International Journal of Applied Biology, 1(1): 1-8.

Gupta, V. K., \& Rastogi, A. 2008. Biosorption of lead from aqueous solutions by green algae Spirogyra species: kinetics and equilibrium studies. Journal of Hazardous Materials, 152(1), 407-414.

Hoerudin \& Irawan B. 2015. The prospect of nanotechnology in building food security. Jakarta (ID): IAARD Press. 49-67 [in Indonesia]

Maruani, I. 2013. Au-Nanoparticle Preparation By Utilizing Green Tea Leaf Extracts For Melamin Pollution Detector. Doctoral dissertation, UPI [In Indonesia]

Özer, A., Akkaya, G., \& Turabik, M. 2006. Biosorption of acid blue 290 (AB 290) and acid blue 324 (AB 324) dyes on Spirogyra rhizopus. Journal of hazardous materials. 135(1): 355364.

Pantidos, N., \& Horsfall, L. E. 2014. Biological synthesis of metallic nanoparticles by bacteria, fungi and plants. Journal of Nanomedicine \& Nanotechnology, 5(5): 1-9.

Qian, H., Pretzer, L. A., Velazquez, J. C., Zhao, Z., \& Wong, M. S. 2013. Gold nanoparticles for cleaning contaminated water. Journal of Chemical Technology and Biotechnology, 88(5), 735-741. 
Rohiman, A., Buchari, M., Juliastuti, E., \& Idris, I. (2014). Synthesis, Characterization, and Applications of Gold Nanoparticles (AuNPs) on the Growth of Silicon Nanowires (SiNWs). Research and Development on Nanotechnology in Indonesia, 1(2), 74. [In Indonesia].

Senapati, S., Syed, A., Moeez, S., Kumar, A., \& Ahmad, A. 2012. Intracellular synthesis of gold nanoparticles using alga Tetraselmis kochinensis. Materials Letters, 79, 116-118.

Shaileyee \& C Mandal, Subhash. 2014. Synthesis And Therapeutic Activity of Silver Nanoparticles Using Bio-Based Protocol. the pharma review.

Sovawi, A. C., Harjono, H., \& Kusuma, S. B. W. (2017). Synthesis of Gold Nanoparticles with Bioreductor Red Guava Fruit Extract (Psidium guajava L.). Indonesian Journal of Chemical Science, 5(3), 169-173 [In Indonesia].

Sulfahri, Mohamad Amin, Sutiman Bambang Sumitro \& Murni Saptasari. 2016. Comparison of biomass production from algae Spirogyra hyalina and Spirogyra peipingensis, Biofuels, 8:3, 359-366.

Thakkar, K. N., Mhatre, S. S., \& Parikh, R. Y. 2010. Biological synthesis of metallic nanoparticles. Nanomedicine: Nanotechnology, Biology and Medicine, 6 (2), 257-262.

Tipnee, S., Ramaraj, R., \& Unpaprom, Y. 2015. Nutritional evaluation of edible fresh water green macroalga Spirogyra varians. Emer. Life Sci. Res, 1, 1-7.

Zarina, A., Masud-ul-Hasan, and Mustafa Shameel. 2007. Diversity Of The Genus Spirogyra (Zygnemophyceae Shameel) In The North-Eastern Areas Of Pakistan. Proc. Pakistan Acad. Sci. 44(4): 225-248.

Zhang, Y., \& Shen, J. 2007. Enhancement effect of gold nanoparticles on biohydrogen production from artificial wastewater. International journal of hydrogen energy, 32(1), 17-23. 